\title{
GROUP REPRESENTATION AND BANACH LIMIT
}

\author{
Masahiro NaKamura ANd Zirô TAKeda
}

(Received August 30, 1950)

1. Introduction. Recently, B. de Sz. Nagy [3] proves the following theorem: In a Hilbert space $H$, any uniformly bounded (cyclic) group of linear operators $T^{n}$ is similar to unitary one, or more precisely, if $\left|T^{n}\right| \leqq k$ for all $n$ then there exists a self-adjoint operator $B$ with $1 / k \leqq B \leqq k$ such that $U=B T B^{-1}$ is unitary. If we consider in this theorem that $T^{n}$ is a "representation" of the additive group of integers in $H$, then we can say that any bounded representation of the group in $H$ is always similar to unitary one. Thus the theorem becomes a generalization of the well-known theorem concerning bounded matrix group-representation. Hence we will consider in this note the above cited de Sz. Nagy's theorem as the following problem :

(A) Is every bounded (strong ly continuons) representation $T(g)$ of a topological group $G$ in a Hilbert space $H$ necessary similar to unitary one?

In $\S 2$, we shall discuss the above problem and partly answer it checking de Sz. Nagy's proof that the problem can be solved if the group $G$ has a Banach limit (Proof can be carried out word for word following to de Sz. Nagy's). Thus the problem (A) is (partly) reduced to the following new problem:

(B) Is it possible to define a Banach limit in the given group G?

This problem will be considered in $\$ 3$, and it is reduced there to a fix-point theorem in the "state" space of all unifomly bounded continuous functions on $G$. Thus it is solved for some special cases which include both abelian and compact.

2. Theorem of B. de Sz. Nagy. Let us assume that $G$ is a topological group and it has a Banach limit $\operatorname{Lim}_{g} x(g)$ for all uniformly bounded continuous functions $x(g)$ on $G$ which satisfies the following conditions :

(1) $\operatorname{Lim}_{g}(\alpha x(g)+\beta y(g))=\alpha \operatorname{Lim}_{g} x(g)+\beta \operatorname{Lim}_{g} y(g)$,

(2) $\quad \operatorname{Lim}_{g} x(g) \geqq 0$ if $x(g) \geqq 0$,

(3) $\operatorname{Lim}_{g} x(g)=1$ if $x(g)=1$ for all $g$,

(4) $\quad \operatorname{Lim}_{g} x(g h)=\lim _{g} x(g)$.

Furthermore, we may assume,that there exists a uniformly bounded strongly continuous representation $T(g)$ of $G$ in a Hilbert space $H$.

Under these circumstances we can solve the problem (A) following to B. de Sz. Nagy [3]. For the sake of completeness we shall repeat it under our notations.

Let $\langle x, y\rangle=\operatorname{Lim}_{g}(T(g) x, T(g) y)$ for any pair $x, y$ of $H$, then $\langle x, y\rangle$ 
becomes bounded hermitian linear form on $H$. Thus by the well-known F. Riesz' theorem we have a self-adjoint positive bounded linear operator $A$ such that $\langle x, y\rangle=(A x, y)$. Using (4) we have also

$$
\begin{aligned}
(A T(g) x, T(g) y) & =\operatorname{Lim}_{h}(T(h) T(g) x, T(h) T(g) y)=\operatorname{Lim}_{h}(T(h g) x, T(h g) y) \\
& =\operatorname{Lim}_{h}(T(h) x, T(h) y)=(A x, y)
\end{aligned}
$$

or, for any $g$ in $G$, we have

$$
\text { (5) } T(g) * A T(g)=A \text {. }
$$

Since it holds $1 / k^{2}|x|^{2} \leqq\langle x, x\rangle \leqq k^{2}|x|^{2}$, we have $1 / k^{2} \leqq A \leqq k^{2}$. Hence for the square root $B$ of $A$ we have $1 / k \leqq B \leqq k$. And so $B^{-1}$ exists. Using (5) we have, for any $g$ in $G$,

$$
\left(B T(g) B^{-1}\right) *(B T(g) B-1)=B^{-1} T(g)^{*} A T(g) B^{-1}=B^{-1} A B^{-1}=\left(B^{-1} B\right)^{2}=I,
$$

whence $B T(g) B^{-1}=U(g)$ is unitary, since $U(g)$ has an inverse.

Summing up the above we have

Theorem 1 (B. DE Sz. NAGY). If $T(g)$ is a uniformly bounded strong ly continuous representation of a topological group $G$ which has a Banach limit satisfying (1)-(4), then there exists a bounded definite selfadjoint operator $B$ such that $U(g)=B T(g) B^{-1}$ is unitary.

Thus we have reduced the problem $(A)$ to $(B)$.

3. Generalized Banach limit. Let $E$ be the Banach space of all realvalued uniformly bounded continuous functions $x(g)$ on $G$ with usual norm $|x|=\sup _{g}|x(g)|$. Furthermore, let $T(g)$ be the "regular right representation" of $G$ on $E$, i. e., $T(g) x(h) \doteq x(h g)$ for all $h$ in $G$. Then obviously $T(g)$ is a positive linear operator on $E$ into itself with norm unity and has an inverse. Hence its conjugate operator $T(g)^{*}$ on $E^{*}$, the conjugate space of $E$, is positive and linear with same norm. Moreover, if $f \in E$ is of positive and norm unity -- a "distribution" or a "state" — then $T(g) * f$ is also. positive and

$$
\begin{aligned}
\| T(g) * f \mid & =\sup \{|T(g) * f(x)| ;|x| \leqq 1\}=\sup \{|f(T(g) x)| ;|x| \leqq 1\} \\
& =\sup \{|f(x)| ;|x| \leqq 1\}=|f|=1
\end{aligned}
$$

since $T(g)$ has an inverse in $E$. Hence $T(g) * f$ is also a state and so $T(g)$. is a "transition operator" in the sense of G. Birkhoff, that is, $T(g) *$ brings a state into a state.

Let now suppose that $D$ be the set of all "states" in $E$, i.e., the set of all $f$ in $E^{*}$ with $f \geqq 0$ and $|f|=f(1)=1$. Then $D$ is evidently bounded and convex, and moreover, it is weak* closed in $E$. Hence by the wellknown theorem of Banach space $D$ is weakly* compact. On the other hand, it is easy to see that $T(g)^{*}$ is weak*-continuous on $D$ and it maps $D$ into $D$, whence by the fix-point theorem of Schauder-Tychonoff $D$ has at least one fix point $f_{0}$ for the given $T(g)$ or $g \in G$. For this state $f_{0}$ and this specific element $g$, if we put $f_{0}(x)=\operatorname{Lim}_{h} x(\boldsymbol{h})$ then we have

$$
\operatorname{Lim}_{h} x(h)=f_{0}(x)=T(g) * f_{0}(x)=f_{0}(T(g) x)=f_{0}(x(h g))=\operatorname{Lim}_{h} x(h g) .
$$

Therefore, the condition (4) holds for all $g$ in $G$ if and only if $f_{0}$ invariants 
under all $T(g)^{*}$. Thus we have proved

THEOREM 2. A topological group $G$ has a Banach limit with (1)-(4) which is defined for all bounded icontinuous functions on $G$, if and only if, there exists an invariant "state" of the space of all such functions under the group of all right-translations.

Thus using the fix-point theorem of A. Markoff and S. Kakutani [2] we have easily from Theorem 2 the following Corollary.

COROLlary 1. Each commutative or soluble group has a Banach limit.

As an immediate consequence of this corollary we have

COROLLARY 2. Each uniformly bounded strongly continuous representation of a commutative or compact topological group in a Hilbert space is always similar to unitary one.

For the compact case, the existence of Banach limit follows from that of invariant mean immediately. Hence the problems (A) and (B) are automatically solved for compact groups.

4. Some remarks. To conclude our note, here we may add some remarks for preceeding section.

A. It may be somewhat interesting to note that the solution of problem (A) for a locally compact abelian group follows from the theory of weak almost periodic functions due to W. F. Eberlein [1]. Since we need essentially in the proof of de Sz. Nagy's theorem that the function

$$
\text { (7) } \quad f(g ; x, y)=(T(g) x, T(g) y), \quad(x, y \in H),
$$

has a "mean" having the properties (1)-(4), the existence of Banach mean for all bounded continuous functions is superfluous. In the case that $G$ is locally compact abelian, we can prove as below that the above cited function is weakly almost periodic in the sense of Eberlien; and so the existence of the mean of weakly almost periodic functions implies that of required functional on the set of all functions of form (7).

To prove that the function ( 7 ) is weakly almost periodic, if we fix $y$ in (7), then (7) defines a bounded linear transformation $T$ from $H$ to $C(G)$ :

$$
T x=(T(g) x, T(g) y) .
$$

Since $|T(g)|$ is uniformly bounded on $G$ by the assumption, $\{T(g) * T(g) x$; $g \in G\}$ is bounded in $H$, whence it is weakly totally bounded in $H$. Moreover, since we have

$$
f(g . h ; x, y) \stackrel{=}{=}(T(h) * T(h) x),
$$

the translates of the function (7) is weakly totally bounded in $C(G)$, that is, (7) is weakly almost periodic.

B. In our proof of Theorem 2, we have excluded the case that the given $G$ is semi-group, and so it excludes automatically the typical case of usual sequential Banach limit: Therefore, here we add a discussion when $G$ is (discrete) semi-group of positive integers.

Suppose that $G$ is a semi-group in the proof of Theorem 2. Since we 
used only the group of $G$ to prove that $T(g) * f$ is a state if $f$ is a state, it suffices to modify this point under some additional condition. If $f$ is a state and all translations $T(g)$ on $E$ keep the constant functians invariant, then $T(g) * f(1)=f(T(g) 1)=f(1)=1$, and so $T(g) * f$ is a state as required.

Therefore, the existence of Banach limit for an abelian semi-group $G$ is a direct consequence of the fix point theorem of Markhoff-Kakutani, if $G$ satisfies the above condition. The typical Banach limit on the natural numbers is of this case.

\section{REFERENCES}

1. W. F.EBeriein, Abstract ergodic theorem and weak almost periodic functions, Trans. Amer. Math. Soc., 67 (1949), 217-240.

2. S. KakUtani, Two fixed-point theorems concerning bicompact convex sets, Proc, Imp. Acad. Tôkyô, 14 (1938), 242-245.

3. B. DE Sz. NAGY, On uniformly bounded linear transformations in Hilbert space, Acta. Szeged., 11(1947), 152-157.

Osaka Normal College aNd TôHokU University

Added in Proof (Aug. 10, 1951)

A. After this note is presented to the Editors, the authors received a letter from $H$. Yoshizawa in which he remarked as follows : (1) If there exists a sequence of compact sets $\left\{C_{n}\right\}$ such that $m\left(C_{n} \div g C_{n}\right) / m\left(C_{n}\right) \rightarrow 0$ for all $g$ of a locally compact group $G$ (where $m$ means the Haar measure and $\div$ symmetric difference), then $G$ has a "Banach limit". (2) An example of a group having no "Banach limit" can be constructed using the method of Hausdorff-von Neumann.

B. ' In the press of this note, recent publications of J. Dixmier (Acta Szeged., 12 (1950), Pars A) and M. M. Day (Trans. Amer. Math. Soc., 69 (1950) ) appeared. Although they handled same material in different manner, their results coincide in many points and also include our above theorems. Our method of proofs is essentially same with that of M. M. Day and $\mathbf{R}$. Godement (the later is quoted in Dixmier's paper). 